\title{
Integrative Gastroenterology and Hepatology Enhanced Role for Apoptosis in a Hepatic Ischaemia-Reperfusion Injury Model
}

\author{
Alzaraa $\mathrm{A}^{\mathbf{1}^{*}}$, Megson $\mathrm{M}^{1}$, Zayyan $\mathrm{K}^{1}$, Morgan $\mathrm{B}^{2}$ and West $\mathrm{K}^{3}$ \\ 'Department of General Surgery, George Eliot Hospital, UK \\ ${ }^{2}$ Departent of Radiology, Leicester Royal Infirmary, UK \\ ${ }^{3}$ Department of Histopathology, Leicester Royal Infirmary, UK \\ *Correspondence: Ahmed Alzaraa, Department of General Surgery, George Eliot Hospital, UK; E-mail: \\ ahmedwahabf@gmail.com
}

Received: May 07, 2018; Accepted: July 26, 2018; Published: July 31, 2018

\begin{abstract}
Background: Prior to reperfusion, a variable period of ischaemia occurs in transplanted donor organs. There is growing evidence that apoptosis contributes to the ischaemia-reperfusion injury (IRI) conundrum that follows, but its extent has not been precisely quantified. Understanding its extent and effects can help find ways to mitigate or counteract such an injury in the liver. We therefore evaluated the extent of apoptosis semi-quantitatively from the expression of Caspase-3 and M30 in a porcine ex-vivo hepatic IRI model and correlated the timing of expression of M30 antibody with that of Caspase-3.

Method: Livers were harvested from three healthy white Landrace Cross pigs and perfused for six hours using oxygenated extracorporeal circuits with heparinized autologous blood at $38^{\circ} \mathrm{C}$. A 2-hour delay prior to perfusion represented the period of ischaemia. All the livers were uniformly perfused. Three biopsies were taken for each of 7 time points (total 21); before dissection (BD) of the livers, after dissection (AD), after connection (AC) to the circuit, at one hour perfused (1 hr P) \& non-perfused (1 hr NP), at 4 hours perfused (4hr P) and non-perfused (4hr NP). Samples were stained with standard Haematoxylin-Eosin (HE) and immunohistochemically with Caspase-3 and M30 monoclonal antibodies. Positive cells were quantified using an ocular grid and expressed as number per square area. Cells Positive for Caspase-3 \& M30 antibodies were counted on the whole section. Data were expressed as median number per square millimeter. Statistical analysis was done using AVOVA and $t$ - test.

Results: After four hours of perfusion, multiple areas of perfusion defects appeared macroscopically in all 3 livers. HE staining confirmed that these non-perfused areas were ischaemic. There was a gradual increase in Caspase-3 expression over time in all samples. There was no statistically significant difference in the Capase-3 and M30 between the 9 specimens at $1 \mathrm{hr} P(p=0.06)$ but there was a highly significant difference between Caspase- 3 and M30 at $1 \mathrm{hr}$ NP $(p=0.001)$. Caspase-3 expression peaked at $4 \mathrm{hr} \mathrm{P}(p=0.001)$ and $4 \mathrm{hr} \mathrm{NP}(p=0.03)$. A similar pattern was noted with M30 which peaked at $1 \mathrm{hr} \mathrm{P}(p=0.001)$ and maintained a non-significant expression at $4 \mathrm{hr} \mathrm{P}(p=0.07)$ and $4 \mathrm{hr} \mathrm{NP}(p$ $=0.1$ ).
\end{abstract}

Conclusion: The significant expression of Caspase-3 and M30 confirms that apoptosis plays an important part in tissue loss in this model of liver ischemia-reperfusion injury. Inhibiting both Caspase-3 and M30 might be useful in alleviating hepatic reperfusion injury.

Keywords: Apoptosis, Ischaemia reperfusion injury, Liver, Animal model, Transplantation Introduction

IRI is a relatively common pathological process that Int Gast Hepatol, 1(1): 48-53 (2018) follows a range of conditions of the liver including trauma, resection, transplantation, infections and shock states 
[1]. IRI occurs when organ ischaemia results in tissue Adenosine Triphosphate (ATP) depletion which leads to the activation of anaerobic metabolic pathways [2]. These do not maintain cellular function for prolonged periods, and that ultimately leads to cell death. Restoration of blood flow is necessary to recover cellular function. Paradoxically however, reperfusion can initiate cascades that cause further cellular damage [3]. Necrosis is the major mode of cell death in IRI, but its occurrence in many other situations such as liver transplantation, haemorrhagic trauma, tumour resection, and many others suggests that other mechanisms of cell death are involved. Recent studies have concluded that apoptosis is another mechanism of cell death in warm and cold hepatic IRI [4]. Dramatic progress has been made over the last two decades in elucidating the intracellular signaling mechanisms of apoptosis [5]. In most cells, a pro-apoptotic mediator binds to its receptor which causes receptor oligomerization and the association of various adapters [4]. However, in hepatocytes, the receptor signal needs to be amplified through the mitochondria [6] which activates Caspases and initiates apoptotic nuclear changes [7]. The apoptosis pathway is activated by the Caspases which are synthesized as inactive precursors and activated by specific cleavage at defined aspartate residues [8].

Herein, we explored the expression of Caspase-3 antibody in IRI to assess the role of apoptosis in IRI. We also used the monoclonal M30 antibody that recognizes a Caspase-generated Cytokeratin-18 neoantigen, and studied its correlation with Caspase-3 in a timedependent manner.

\section{Method}

\section{Porcine livers}

Three white Landrace Cross pigs weighing $45 \mathrm{~kg}, 50 \mathrm{~kg}$ and $60 \mathrm{~kg}$ were humanely sacrificed in accordance with Home Office regulations and autologous blood was collected into a container with 5,000 units of heparin. The livers were retrieved according to the technique previously described [9-11], and 2L of cold Soltran solution (Baxter Healthcare, Thetford, UK) at room temperature were perfused, $1 \mathrm{~L}$ into the Portal Vein (PV) and $1 \mathrm{~L}$ into the Hepatic Artery (HA). After the cold perfusion, the livers were transported on ice to the laboratory.

\section{The liver model}

Using autologous blood, the liver perfusion was maintained via the extracorporeal circuit similar to that used for cardiopulmonary extracorporeal bypass surgery
(Medtronic Inc., Minneapolis, Minnesota, USA) [12]. It consists of an automatic centrifugal pump which provided the hepatic arterial flow and pressure, an oxygenator, a heat exchanger unit and a blood reservoir to simulate the venous flow and pressure.

\section{Circuit preparation}

Autologous heparinized blood was used to prime the circuit during the backbench preparation. Cannulation of the Portal Vein (PV), Hepatic Artery (HA), bile duct, supraand infra-hepatic Inferior Vena Cava (IVC) was carried out during the priming. Before connecting the liver to the circuit, 1 liter of $0.9 \%$ normal saline solution (Baxter Healthcare, Thetford, UK) was perfused through the PV and HA to flush out the Soltran solution and remove air from the organ and the cannulae.

\section{Perfusion}

The venous blood was collected from the supra and infra-hepatic IVC and returned to the centrifugal pump. Parenteral nutrition, vasodilating prostacyclins, sodium bicarbonate, sodium taurocholate a single dose of Augment in (1.2g) and insulin were added to the circulation to optimize the physiological condition of the system with dosages and rates of infusions similar to previous protocols [13]. Perfusion was carried out for $6 \mathrm{~h}$ and haemodynamic parameters (HA and PV pressures and flows) were recorded hourly.

\section{Specimens}

Histological examination: Three biopsies were chosen for each time point (total 21); Before Dissection (BD) of the livers, After Dissection (AD), After Connection (AC) to the circuit, at one hour perfused (1hr P) \& nonperfused ( $1 \mathrm{hr} \mathrm{NP})$, at 4 hours perfused $(4 \mathrm{hr} P)$ and non-perfused (4hr NP) \& analyzed with standard Haematoxylin-Eosin staining. Histological assessment of the extent of liver injury was performed using the Suzuki classification [14] which grades injury from 0-4 as illustrated in table 1. Immunohistochemical staining was performed on the biopsies with Caspase-3 and M30 monoclonal antibodies.

Antigen retrieval: In order to retrieve the antigens, the specimens were cut onto Vectabond slides, left to dry at $37^{\circ} \mathrm{C}$ overnight and heated for 10 minutes at $65^{\circ} \mathrm{C}$. They were then deparaffinised using xylene, rehydrated using graded alcohols $(99 \%$ then $95 \%$ Industrial Methylated Spirits "IMS") and then rinsed with tap water for five minutes. The slides were placed on a slide rack in a plastic dish, which was then topped up with $10 \mathrm{mM}$ 
Sodium Citrate ( $\mathrm{pH}$ 6.0). The dish was micro waved at $80 \%$ power for 20 minutes and left to cool in the buffer at room temperature.

Table 1: Suzuki classification for the histological assessment of the extent of liver injury [14].

\begin{tabular}{c|c|c|c}
\hline $\begin{array}{c}\text { Numerical } \\
\text { assessment }\end{array}$ & $\begin{array}{c}\text { Sinusoidal } \\
\text { congestion }\end{array}$ & $\begin{array}{c}\text { Vacuolation/ } \\
\text { ballooning }\end{array}$ & Necrosis \\
\hline 0 & None & None & None \\
\hline 1 & Minimal & Minimal & Single cell \\
\hline 2 & Mild & Mild & $<30 \%$ \\
\hline 3 & Moderate & Moderate & $30 \%-60 \%$ \\
\hline 4 & Severe & Severe & $>60 \%$ \\
\hline
\end{tabular}

\section{Immunohistochemistry assays:}

Immunohistochemistry for apoptosis was performed using Cleaved Caspase-3 "Asp 175", (New England Biolabs) as the primary antibodies: and M30 (CytoDeath. Bioaxxess) utilizing NovoLink Polymer Detection System (Leica Microsystems. RE7140-CE). The slides were washed with a buffering Blocking Solution - (TBS/3\%BSA/0.1\% Triton-X-100) which acted as the primary antibody diluents. The endogenous peroxidase was neutralized by using Peroxidase Block for 5 minutes. The slides were washed in alternate cycles using TBS for $2 \times 5$ minutes. After each washing cycle, the following was done in this order: the slides were incubated with Protein Block for 5 minutes, incubated with optimally diluted primary antibody, second incubation with Post Primary Block for 30 minutes, third incubation with NovoLink Polymer for 30 minutes and finally developed peroxidase activity with DAB working solution for 5 minutes. The slides were then washed in tap water for another 5 minutes and counterstained using Mayer's Haematoxylin for 30 seconds. A final wash with tap water for 5 minutes before dehydration with air and mounted in DPX. The identification of apoptotic cells was confirmed when there was evidence of Caspase-3 and M30 positivity.

Quantitative apoptotic index: The apoptotic index was expressed as the ratio of the number of hepatic cells with Caspase-3 and M30 positivity out of the total number of nucleated cells in each field (magnification, $\times 40$ ) calculated after counting 5 random microscopic fields for each time point with a $19 \mathrm{~mm}$ Whipple grid graticulelens (Pyser-SGI LTD). In each field, 100 squares were evaluated for the presence of apoptotic cells. Activated Caspase-3 hepatocytes positive for DNA fragmentation (Figure 1) and for cytoplasmic activity for M30 (Figure 2) were counted on the whole sections. The mean counts were expressed as a percentage of the total number of nonapoptotic cells counted in each field.

\section{Results}

\section{Microscopic examination}

Caspase-3 expression: Caspase-3 maintained the same expression $B D \& A D$, dipped $A C$ then gradually increased after perfusion in the Perfused and nonperfused areas (Figure 2).

M30 expression: There was not much evidence of M30 BD. It slightly increased $A D$, dipped $A C$, but peaked at $1 \mathrm{hr}$ P. Its expression was less in the $1 \mathrm{hr} \mathrm{NP}$ and at $4 \mathrm{hr}$ $\mathrm{P}$ and $4 \mathrm{hr}$ NP (Figure 3).

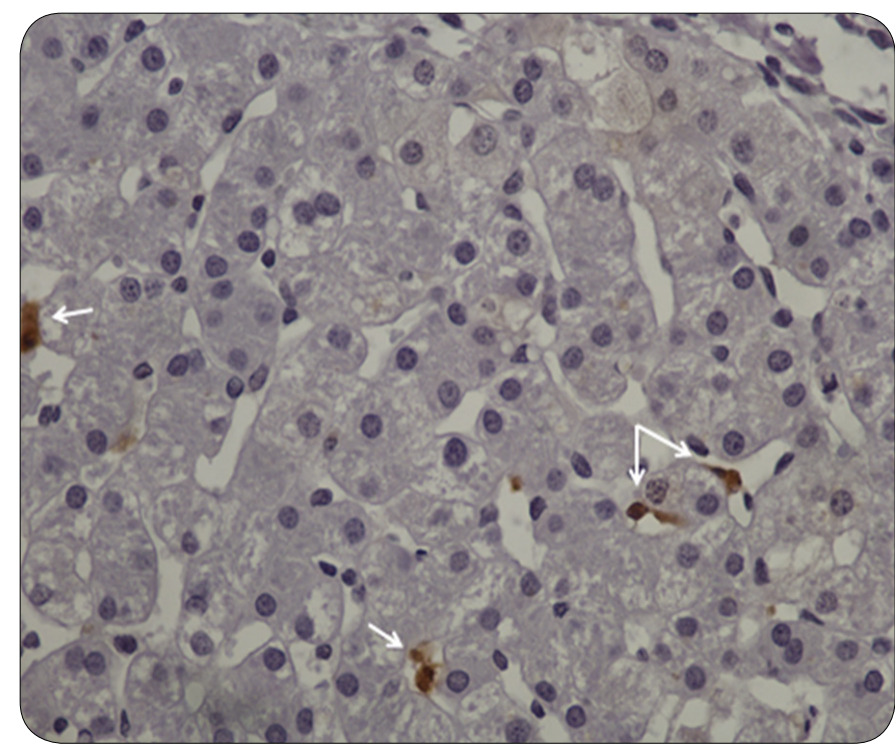

Figure 1: An example of Caspase-3 expression "White arrows" (×50).

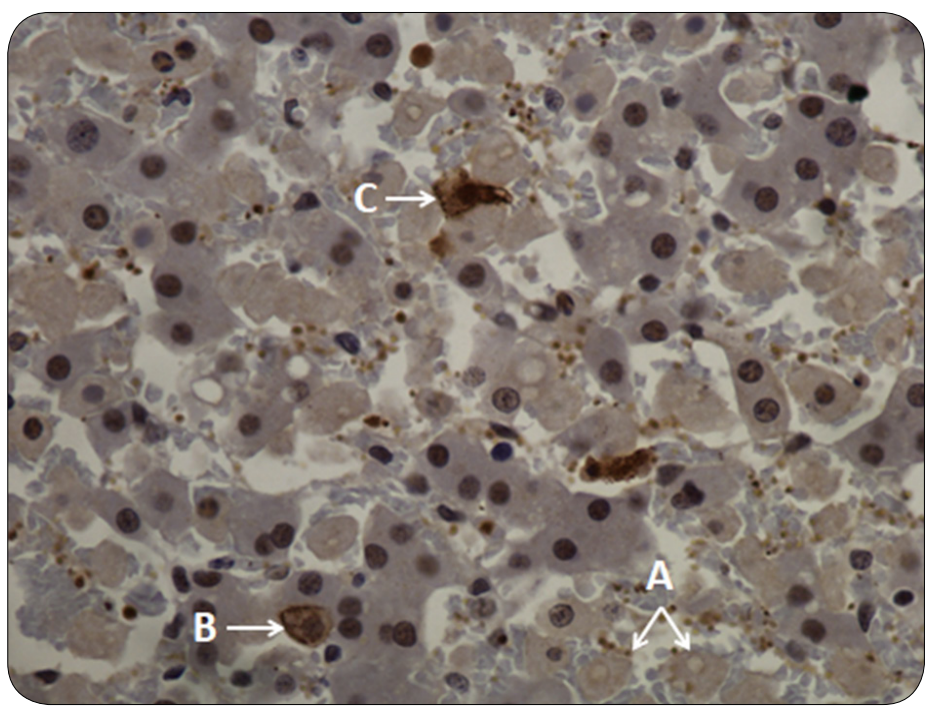

Figure 2: An example of M30 expression in hepatocytes ranging from weak $(A)$, intermediate $(B)$ to 
strong $(C)$ reactions (original magnification $\times 50$ ).

\section{Statistical analysis}

Median values of the different groups were compared by ANOVA and linear correlation tests (Table 2 ).

Caspase-3 expression: There was a gradual increase in Caspase- 3 expression in all samples. The difference was non-significant at $1 \mathrm{hr} \mathrm{P}(p=0.06)$ and a highly significant at $1 \mathrm{hr} \mathrm{NP}(p=0.001)$, then it peaked at $4 \mathrm{hr} \mathrm{P}(p=0.001)$ and $4 \mathrm{hr} \mathrm{NP}(p=0.03)$ (Figure 4).

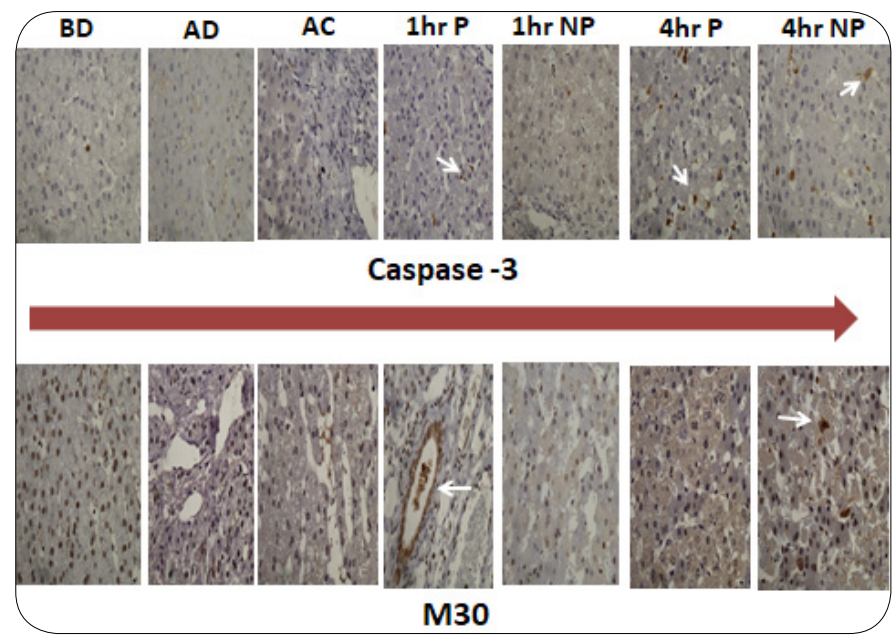

Figure 3: A comparison between the expression of Caspase-3 (upper panel) \& M30 (lower panel) "white arrows" at each point in time $(\mathrm{BD}=$ Before Dissection, $A D=$ After Dissection, $A C=$ After Connection, $1 \mathrm{hr} P=1$ hour

Perfused, $1 \mathrm{hr} N \mathrm{~N}=1$ hour Non-Perfused, 4hr $\mathrm{P}=4$ hour

Perfused, 4hr NP=4hour Non-Perfused) (Original magnification $\times 50$ ).

M30 expression: A similar pattern to Caspase-3 expression was noted with M30 which peaked at $1 \mathrm{hr} \mathrm{P}(p$ $=0.001)$ and maintained a non-significant expression at $4 \mathrm{hr} P(p=0.07)$ and $4 \mathrm{hr} \mathrm{NP}(p=0.1)$. The overall difference among the groups along the time scale was significant ( $p$ $=0.01$ ) (Figure 4).

\section{Discussion}

Its dual blood supply makes the liver uniquely suitable for IRI modeling. The complex conundrum of IRI in the liver appears to involve cellular mediators, reactive oxygen species, the compliment system, cytokines and other secreted factors. It has been postulated that much of the damage in IRI was due to necrosis [5]. However there is growing evidence that apoptosis also contributes to the damage since blocking necrosis does not completely stop the damage from IRI [5].

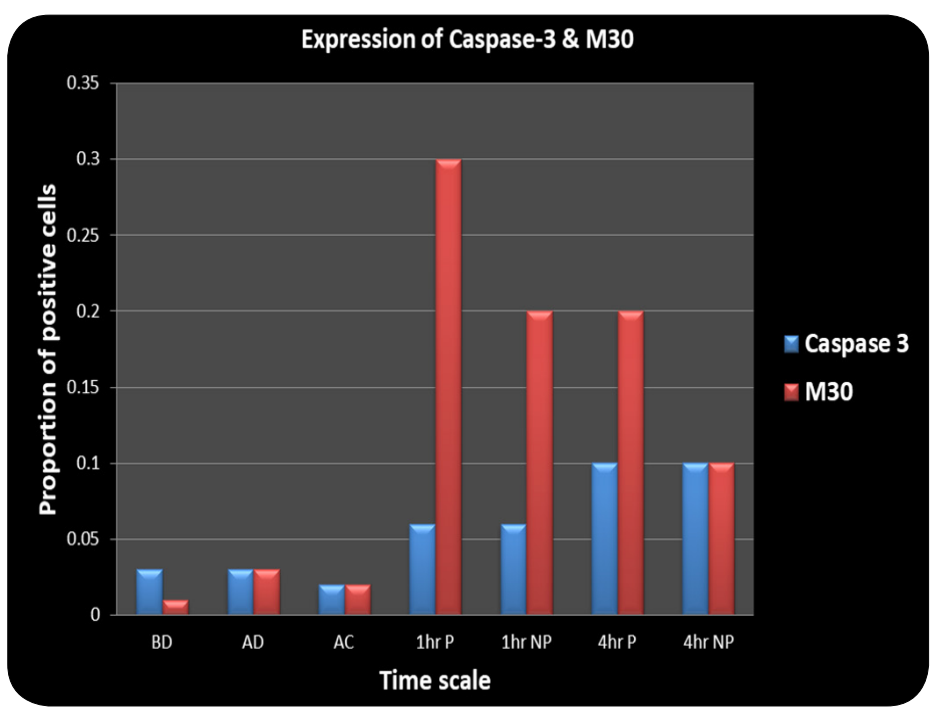

Figure 4: Expression of Caspase-3 \& M30 in the liver biopsies at each time point $(B D=$ Before Dissection, $A D$ $=$ After Dissection, $\mathrm{AC}=$ After Connection, $1 \mathrm{hr} \mathrm{P}=1$ hour Perfused, $1 \mathrm{hr}$ NP $=1$ hour Non-Perfused, $4 \mathrm{hr} \mathrm{P}=4$ hour Perfused, 4 hr NP $=4$ hour Non-Perfused).

None of the proxy methods currently used to infer apoptosis or its extent is considered to be valid. For example, serum parameters such as ALT, AST and LDH do not reflect the scale of IRI tissue injury as they have to be monitored before and after perfusion [15]. Although HE staining demonstrates Councilman Bodies which are

Table 2: Data from the three experiments showing the Mean and Standard Deviation (SD) of the proportions of positive cells that stained positive for Caspase-3 and M30 at different time points $(B D=$ Before Dissection, $A D=A f t e r$ Dissection, AC = After Connection, $1 \mathrm{hrP}=1$ hour Perfused, $1 \mathrm{hrNP}=1$ hour Non-Perfused, $4 \mathrm{hrP}=4$ hour Perfused, 4 hrNP $=4$ hour Non-Perfused).

\begin{tabular}{l|c|c|c|l|l|l|l}
\hline Caspase-3 & BD & Ad & AC & 1hr P & 1hr NP & 4hr P & 4hr NP \\
\hline Mean & 0.03 & 0.03 & 0.02 & 0.06 & 0.06 & 0.1 & 0.1 \\
\hline SD & 0.02 & 0.03 & 0.02 & 0.02 & 0.01 & 1.6 & 0.06 \\
\hline M30 & & & & & & & \\
\hline Mean & 0.01 & 0.03 & 0.02 & 0.3 & 0.2 & 0.2 & 0.1 \\
\hline SD & 0.01 & 0.02 & 0.01 & 0.09 & 0.1 & 0.1 & 0.1 \\
\hline
\end{tabular}


the end result of apoptosis, it does not reflect the number of events in this mode of cell death [16]. The terminal deoxynucleotidyl transferase-mediated deoxyuridine triphosphate nick-end labeling (TUNEL) assay is no different from the first two methods. It is not reliable as it has been shown to be non-specific. It requires highly standardized procedures and it does not differentiate between apoptotic and necrotic cells $[16,17]$.

The exploration of different modes of cell death can help open new venues for therapeutic targeting of IRI. Jaeschke et al. [5] propose the theory of necroapoptosis in which common pathways lead to both necrosis and apoptosis. This is due to the mitochondrial permeability transition, whereby ATP depletion leads to cell membrane failure and necrosis while partial ATP preservation allows the mitochondria to release Caspases which lead to apoptosis. Therefore in this theory, necrosis and apoptosis could be thought of as leading to the ends of a spectrum depending on the ATP depletion. Accordingly if the start of this common pathway could be found then both necrosis and apoptosis could be reduced during liver IRI.

In this study, we have demonstrated the correlation between Caspase-3 and M30 antibodies in IRI- associated apoptosis, showing a statistically significant gradual increase in Caspase-3 expression, However M30 showed a statistical significant increase after the first hour. Those results show that apoptosis as well as necrosis had a significant role to play in liver IRI. Therefore to stop the injury from IRI then one would need to stop both the pathways of necrosis and apoptosis.

\section{Limitations of the study}

Ex-vivo models like this one are limited by a 6-hour run time in contrast to in-vivo models such as that of Sun, et al. [18] and Tsung et al. [19] who could extend their experiment to measure late effects for up to $24 \mathrm{hrs}$ after reperfusion [18]. Secondly, the accuracy of the apoptotic index depends on the identification of apoptotic cells which can be magnification dependent, as Shining et al. [20] point out. To reduce such counting errors we used the recommended high-power $(\times 40)$ lens. Finally, because the duration of morphologically detectable stage of apoptosis may vary, apoptotic index does not always correlate well with an actual "death index" [21].

\section{Conclusion}

In this model, apoptosis as measured by Caspase-3 and M30 expressions plays a significant role in liver IRI.
However, further research is needed to validate this both ex-vivo and in-vivo to confirm our findings and thus pave the way for work to find a suitable apoptosisbased therapeutic target. This could have wide ranging implications and application in liver trauma, sepsis, resection and transplantation.

\section{Author's Contribution}

Ahmed Alzaraa: Principal investigator, conducted the experiments, drafted and edited the article.

Matthew Megson: Literature research, editing of the article.

KassimZayyan: Revised and edited the article.

Bruno Morgan: Study Planning and Manuscript editing. Kevin West: Edited the histology analysis.

Competing interests: The authors declare that there are no competing interests.

Approval: All authors have approved the article.

\section{References}

1. Grace PA. Ischaemia-reperfusion injury. British Journal of Surgery. 1994;81(5):637-647. doi: http://dx.doi. org/10.1002/bjs.1800810504

2. Carden DL, Granger DN. Pathophysiology of ischaemia-reperfusion injury. The Journal of Pathology. 2000;190(3):255-266. doi: http://dx.doi. org/10.1002/(SICI)1096-9896(200002)190:3<255::AIDPATH526>3.0.CO;2-6

3. Datta G. Molecular mechanisms of liver ischemia reperfusion injury: Insights from transgenic knockout models. World Journal of Gastroenterology. 2013;19(11):1683. doi: http://dx.doi.org/10.3748/wjg. v19.i11.1683

4. Jaeschke $\mathrm{H}$, Lemasters JJ. Apoptosis versus oncotic necrosis in hepatic ischemia/reperfusion injury. Gastroenterology. 2003;125(4):1246-1257. doi: http:// dx.doi.org/10.1016/S0016-5085(03)01209-5

5. Peter ME, Krammer PH. Mechanisms of CD95 (APO1/Fas)-mediated apoptosis. Current Opinion in Immunology. 1998;10(5):545-551. doi: http://dx.doi. org/10.1016/S0952-7915(98)80222-7

6. Scaffidi C. Two CD95 (APO-1/Fas) signaling pathways. The EMBO Journal. 1998;17(6):1675-1687. doi: http:// dx.doi.org/10.1093/emboj/17.6.1675

7. Yin X-M. Bid, a critical mediator for apoptosis induced by the activation of Fas/TNF-R1 death receptors in hepatocytes. Journal of Molecular Medicine. 
2000;78(4):203-211. doi: http://dx.doi.org/10.1007/ s001090000099

8. Gravante G, Ong SL, West $\mathrm{K}$, et al. Patterns of Histological Changes following Hepatic Electrolytic Ablation in an Ex-Vivo Perfused Model. Pathology \& Oncology Research. 2012;18(4):1085-1089. doi: http:// dx.doi.org/10.1007/s12253-012-9549-1

9. Cursio R. Caspase inhibition in liver transplantation: from basic research to clinical studies. $H P B$. 2010;12(1):1-3. doi: http://dx.doi.org/10.1111/j.14772574.2009.00123.x

10. Gravante G, Ong SL, Metcalfe MS, Lloyd DM, Dennison AR. The Porcine Hepatic Arterial Supply, Its Variations and Their Influence on the Extracorporeal Perfusion of the Liver. Journal of Surgical Research. 2011;168(1):5661. doi: http://dx.doi.org/10.1016/j.jss.2009.09.050

11. Gravante G, Ong SL, Metcalfe MS, et al. Effects of Hypoxia Due to Isovolemic Hemodilution on an Ex Vivo Normothermic Perfused Liver Model. Journal of Surgical Research. 2010;160(1):73-80. doi: http:// dx.doi.org/10.1016/j.jss.2008.09.024

12. Alzaraa A, Al-Leswas D, Chung WY, et al. Contrastenhanced ultrasound detects perfusion defects in an ex vivo porcine liver model: a useful tool for the study of hepatic reperfusion. Journal of Artificial Organs. 2013;16(4):475-482. doi: http://dx.doi.org/10.1007/ s10047-013-0717-1

13. Chung WY, Gravante G, Al-Leswas D, et al. The autologous normothermic ex vivo perfused porcine liver-kidney model: improving the circuit's biochemical and acid-base environment. The American Journal of Surgery. 2012;204(4):518-526. doi: http://dx.doi. org/10.1016/j.amjsurg.2011.11.016

14. Ke B, ShenX-D, Lassman CR, Gao F, Busuttil RW, KupiecWeglinski JW. Cytoprotective and Antiapoptotic Effects of IL-13 in Hepatic Cold Ischemia/Reperfusion Injury Are Heme Oxygenase-1 Dependent. American Journal of Transplantation. 2003;3(9):1076-1082. doi: http://dx.doi.org/10.1034/j.1600-6143.2003.00147.x

15. Mas VR. Ischemia/reperfusion, does temperature matter? Laboratory perspective. Liver Transplantation.
21(S1):S1-S5. doi: http://dx.doi.org/10.1002/lt.24322

16. Nayak A, Raikar A, Kotrashetti V, Nayak R, Shree S, Kambali S. Histochemical detection and comparison of apoptotic cells in the gingival epithelium using hematoxylin and eosin and methyl green-pyronin: A pilot study. Journal of Indian Society of Periodontology. 2016;0(0):0. doi: http://dx.doi.org/10.4103/0972124X.182601

17. Grassi A, Susca M, Ferri $S$, et al. Detection of the M30 Neoepitope as a New Tool to Quantify Liver Apoptosis Timing and Patterns of Positivity on Frozen and Paraffin-Embedded Sections. American Journal of Clinical Pathology. 2004;121(2):211-219. doi: http:// dx.doi.org/10.1309/UK62-1LFJ-4FX0-7KDE

18. Sun K. Role of mitochondria in cell apoptosis during hepatic ischemia-reperfusion injury and protective effect of ischemic postconditioning. World Journal of Gastroenterology. 2004;10(13):1934. doi: http://dx.doi. org/10.3748/wjg.v10.i13.1934

19. Tsung A, Sahai $R$, Tanaka $H$, et al. The nuclear factor HMGB1 mediates hepatic injury after murine liver ischemia-reperfusion. The Journal of Experimental Medicine. 2005;201(7):1135-1143. doi: http://dx.doi. org/10.1084/jem.20042614

20. Soini Y, Pääkkö P, Lehto V-P. Histopathological Evaluation of Apoptosis in Cancer. The American Journal of Pathology. 1998;153(4):1041-1053. doi: http://dx.doi.org/10.1016/S0002-9440(10)65649-0

21. Maione F, Gilbo N, Lazzaro S, et al. Porcine Isolated Liver Perfusion for the Study of Ischemia Reperfusion Injury: A Systematic Review. Transplantation. 2018;102(7):1039-1049. doi: http://dx.doi. org/10.1097/TP.0000000000002156

Copyright: (c) Alzaraa et al. This is an Open Access article distributed under the terms of the Creative Commons Attribution License, which permits unrestricted use, distribution, and reproduction in any medium, provided the original work is properly cited. 